\title{
Viral neuroimmunology/Viral neuropathogenesis I
}

\author{
Chairpersons: R. Fujinami (Salt Lake City, USA) \\ T. Weber (Hamburg, D)
}

31

\author{
Encephalitis and demyelination by murine \\ coronaviruses: early determinants of disease \\ M.J. Buchmeier \\ The Scripps Research Institute (La Jolla, USA)
}

Viruses are among the most common and ubiquitous environmental toxins encountered in the course of the normal human life span. Neurotropic viruses are encountered both as circulating epidemic agents as well as in the context of predominantly non-neurological exposure. The outcome of these infections may vary widely from frank encephalitis, paralytic poliomyelitis or other catastrophic outcome, to inapparent infections. The principal factor that controls the outcome of infection is the host response. It is the role of the host response to contain and control the spread of viral infection in the CNS, and paradoxically, this response may itself be pathological. The ability of a virus to manipulate the immune system has a pivotal effect on the progression of disease and the fate of the infected host. Using two murine coronaviruses, MHV-A59 and MHV-JHM as models for viral-induced demyelination and lethal encephalitis respectively, the contribution of virally-induced immune responses to disease was evaluated. Distinct glial cell cytokine and chemokine responses were observed in infected brains soon after infection, and as the disease progressed, peripheral immune cells became increasingly involved, these responses became increasingly polarized. MHV-A59 infection triggered early and sustained upregulation of IFNg mRNA that was accompanied by a 3 -fold increase in the infiltration of CD8 T cells compared to MHV-JHM infected brains. MHV-A59 infected mice also exhibited increased numbers of IFNg producing cells during acute encephalitis. In contrast, MHV-JHM infection induced strong and ongoing transcription of the innate immune products IFNb, IL-6, and IL-1. High levels of MIP-1 and MIP-2 message observed at the onset of MHV-JHM infection correlated with a marked elevation in the number of macrophages and neutrophils in the brain relative to MHV-A59 infection. Our results indicate that the fate of neurotropic viral infections may be determined by early resident brain-cell innate responses and their effect on the preferential accumulation of peripheral cell populations within the CNS.
32

The cellular immune response against JC virus in progressive multifocal leukoencephalopathy I. Koralnik, R. Du Pasquier, M. Kuroda, J. Schmitz, X. Dang, Y. Zheng, M. Lifton, N. Letvin

Harvard Medical School (Boston, USA)

Progressive Multifocal Leukoencephalopath y (PML) is a demyelinating disease of the central nervous system caused by a reactivation of the polyomavirus JC (JCV) in the setting of immunosuppression. We have explored JCV-specific cellular immune response in patients with PML and control subjects. HIV+/PML survivors, but not HIV+/PML progressors had CD8+ cytotoxic T Lymphocytes (CTL) against either the JCV T regulatory protein or the VP1 major capsid protein. $(1,2)$. CTL recognize virus-infected cells through the interaction of their T-cell receptor with an 8-10 amino-acid viral epitope in association with an MHC class I molecule on the surface of the infected cells. To facilitate more precise studies of JCV-specific CTL, we have chosen to characterize the JCV epitope peptides presented to CTL by the commonly expressed MHC class I molecule HLA-A*0201. We identified a 9 amino-acid epitope of the VP1 protein, the VP1p100 peptide ILMWEAVTL, which is recognized by CTL of HLA-A2+ HIV+/PML survivors. We then constructed an HLA-A*0201/VP1p100 tetrameric complex which allowed us to assess by flow cytometry the PBMC of 13 PML patients and 11 control subjects for the presence of JCV-specific CTL. VP1p100-specific CTL were detected by tetramer binding in VP1p100-stimulated PBMC of 5/7 (71\%) PML survivors and 0/6 PML progressors $(p=0.02)$. Two of 3 HIV+ patients with a leukoencephalopath y resembling PML but with no virologic evidence of JCV infection also had detectable VP1p100specific CTL in their PBMC. PBMC of $8 \mathrm{HIV}+$ patients with other neurologic diseases and healthy control subjects had no detectable JCV-specific CTL (3). These data suggest that the JCV-specific cellular immune response may be important in the containment of PML, and the tetramer staining assay may provide a useful prognostic tool in the clinical management of these patients.

This work was supported by NIH grant NS01919 and NS/AI41198, the Harvard Center For AIDS Research grant P30-AI28691 and a Milton Fund grant to IJK.

1. Koralnik, et al. (2001). J Virol 75: 3483-3487.

2. Du Pasquier, et al. (2001). J. Neurovirol. 7: 318-322.

3. Koralnik, et al. (2002). J. Immunol., in press. 
33

\section{Host genetic control of Theiler's virus persistent infection and demyelinating disease}

M. Brahic, J.-F. Bureau

Institut Pasteur (Paris, FR)

Theiler's virus, a murine picornavirus, causes a persistent infection of the CNS accompanied by focal inflammation and primary demyelination. This disease is a model for multiple sclerosis. Inbred strains of mice vary greatly in their susceptibility to the disease. This is due to a multigenic control in which the H-2D gene plays a major role. Non $\mathrm{H}-2$ susceptibility genes have been mapped by scanning the genome of an $($ SJL/J $\times$ B10.S $) \times$ B10.S backcross. Susceptibility loci have been found close to the Ifng locus on chromosome 10. A series of $\mathrm{H}-2 \mathrm{~s}$ mice congenic for the chromosome 10 region has been constructed and characterized. Their analysis showed that Ifng which codes for interferon gamma, is not a susceptibility locus. Instead, two susceptibility loci, Tmevp2 and Tmevp3, have been located in the region. The mechanism by whichTmevp 3 controls viral persistence has been investigated in detail. Experiments using lethal irradiation followed by bone marrow reconstitution showed that hematopeietic cells are responsible for the difference of susceptibility between the SJL/J and B10.S strains. The effect of theTmevp3 locus depends also on subtle variations on the viral genome as shown with a line of congenic mice in which the B10.S allele of the locus has been isolated on an SJL/J background. A candidate gene for Tmevp3, which is expressed in peripheral lymphocytes, has been identified and characterized.

Studying combinations of parental and congenic mice inoculated with two different variants of Theiler's virus strain DA showed that only mice with a high load of persisting viral RNA in CNS developed clinical signs of demyelination, as determined with the Rotarod test. However, high viral load is not sufficient and in some of the combinations mice with high viral load were resistant to clinical disease. A locus on chromosome 11 (lod score $=4.8$ ), which affects the severity of clinical disease for mice with a high viral load has been identified.

\section{4}

Semliki Forest virus pathogenesis

J. Fazakerley

University of Edinburgh (Edinburgh, UK)

In mice, the outcome of infection by the alphavirus Semliki Forest virus (SFV) varies according to age of the mouse and strain of the virus and can include acute encephalitis, subacute demyelinating meningoencephalomyelitis and persistent subclinical CNS infection. All strains of the virus are virulent in mice infected at less than 12 days of age. The L10 strain is also virulent in mice $>12$ days age whereas the A7(74) strain is avirulent in mice infected at older than 14 days of age. The genetic difference between the two strains of virus maps to the nsp3 gene, though there are a number of loci scattered throughout the genome which affect neurovirulence. For A7(74) virus, age-related virulence correlates with ability of CNS neurones to replicate virus and undergo apoptotic cell death. Immature developing neurones support complete virus replication but as neuronal populations mature virus infection becomes restricted and non-productive. Infection of developing neuronal populations results in apoptosis whereas infection of mature neurones results in persistent infection. The signalling mechanism for the induction of apoptosis upon infection has not been defined but an intact type-I interferon system and a functional PKR gene are not required. The type I interferon system is crucial for protection of adult mice against avirulent strains of SFV. Neither PKR nor RNAse-L, both interferon inducible genes are required for this protective effect, though PKR activity contributes to virus clearance from the brain. An active type-I interferon system prevents virus spread in extraneural tissues, particularly in the pancreas and prevents virus replication in meningeal and ependymal cells in the brain. Absence of this system does not relieve the restricted virus replication observed in mature neurones. This restricted replication can be overcome by gold compounds which may function by inducing neuronal dedifferentiation to a state permissive for virus replication. 